\title{
Chloride Channels and Endocytosis: New Insights from Dent's Disease and CIC-5 Knockout Mice
}

\author{
Olivier Devuyst François Jouret Céline Auzanneau Pierre J. Courtoy \\ Division of Nephrology; CELL Unit, Christian de Duve Institute of Cellular Pathology, \\ Université catholique de Louvain Medical School, Brussels, Belgium
}

\author{
Key Words \\ CLC knockout mice - Fanconi syndrome, renal • \\ Endosomal acidification - Proteinuria, low-molecular \\ weight
}

\begin{abstract}
Dent's disease is a hereditary renal tubular disorder characterized by low-molecular weight (LMW) proteinuria, hypercalciuria and nephrolithiasis. The disease is due to mutations of CIC-5, a member of the family of voltage-gated CLC chloride channels. CIC- 5 is expressed in part in cells lining the proximal tubule (PT) of the kidney, where it colocalizes with albumin-containing endocytic vesicles belonging to the receptor-mediated endocytic pathway that ensures efficient reabsorption of ultrafiltrated LMW proteins. Since progression along the endocytic apparatus requires endosomal acidification, it has been suggested that dysfunction of $\mathrm{CIC}-5$ in endosomes may lead to inefficient reabsorption of LMW proteins and dysfunction of PT cells. Analysis of a CIC-5 knockout (KO) mouse model, displaying all the characteristic renal tubular defects of Dent's disease, showed evidence of a severe LMW proteinuria. Cytochemical studies with the endocytic tracer, peroxidase, showed poor transfer into early endocytic vesicles, suggesting that impairment of receptor-mediated endocytosis in PT cells is the basis for the defective uptake of LMW proteins in pa-
\end{abstract}

Based on a presentation at the 71st Congress of the French Society of Physiology in Paris, September 2004.
(C) 2005 S. Karger AG, Basel

$1660-2137 / 05 / 0993-0069 \$ 22.00 / 0$

Fax +4161306 1234 E-Mail karger@karger.ch www.karger.com
Accessible online at:

www.karger.com/nep tients with Dent's disease. Endocytosis and processing of LMW proteins involve the multiligand tandem receptors, megalin and cubilin, that are abundantly expressed at the brush border of PT cells. Characterization of the endocytic defect in CIC-5 KO mice revealed that ligands of both megalin and cubilin were affected. The total kidney content of megalin and especially cubilin at the protein level was decreased but, more importantly, using analytical subcellular fractionation and quantitative immunogold labelling we demonstrated a selective disappearance of megalin and cubilin at the brush border of PT cells. These observations allowed us to conclude that defective protein endocytosis linked to $\mathrm{CIC}-5$ inactivation is due at least in part to a major and selective loss of megalin and cubilin at the brush border, reflecting a trafficking defect in renal PT cells. These results improve our understanding of Dent's disease, taken as a paradigm for renal Fanconi syndrome and nephrolithiasis, and demonstrate multiple roles for $\mathrm{CIC}-5$ in the kidney. These studies also provided insights into important functions such as apical endocytosis, handling of proteins by renal tubular cells, calcium metabolism, and urinary acidification.

Copyright $(2005$ S. Karger AG, Basel

\section{Introduction}

Kidney stones (nephrolithiasis) represent a major health problem as they affect more than $10 \%$ of males and $5 \%$ of females by the age of 70 years and are often com-

Olivier Devuyst

Division of Nephrology, UCL Medical School

10 Avenue Hippocrate

B-1200 Brussels (Belgium)

Tel. +32 276418 55, Fax +32 276454 55, E-Mail devuyst@nefr.ucl.ac.be 
plicated by nephrocalcinosis and renal failure. Nephrolithiasis occurs as an inherited disorder in about half the patients and is most commonly associated with hypercalciuria. The biochemical basis for hereditary nephrolithiasis and hypercalciuria is unknown, which has hampered therapeutic developments [1].

Recent genetic studies have provided important clues to the understanding of nephrolithiasis. Three disorders of hypercalciuric nephrolithiasis (Dent's disease and its variants X-linked recessive nephrolithiasis and X-linked recessive hypophosphatemic rickets) have been mapped to Xp11.22 [2]. These disorders are characterized by a dysfunction of the renal proximal tubule (PT; renal Fanconi syndrome), with low-molecular weight (LMW) proteinuria, hypercalciuria, nephrocalcinosis, nephrolithiasis and renal failure [3]. A search for renally expressed genes from this region identified inactivating mutations of CLCN5, a member of the CLC family of voltage-gated chloride channels, in Dent's disease and its variants [4]. Despite these genetic advances, the mechanisms whereby the loss of a chloride channel leads to hypercalciuria and renal tubular defects remain unknown.

This review describes a series of molecular investigations that allowed us to investigate the pathophysiology of Dent's disease taken as a paradigm of renal tubular disease and nephrolithiasis. These studies provided new insights into the role of chloride channels in apical endocytosis and renal tubular handling of proteins, calcium and protons.

\section{Intrarenal and Subcellular Distribution of CIC-5 Suggests a Pathophysiological Basis for Dent's Disease}

CLCN5 belongs to the CLC family of voltage-gated chloride channel genes, of which nine different members have been identified in mammals. These chloride channels are important for the control of membrane excitability, transepithelial transport, and possibly regulation of cell volume [5]. CLCN5 is predominantly expressed in the kidney. It encodes $\mathrm{ClC}-5$, a 746-amino acid protein that induces strong outwardly rectifying chloride currents in heterologous expression systems. These currents are markedly reduced or abolished by mutations of Dent's disease [3-5].

In order to understand how the loss of the chloride channel ClC-5 leads to multiple renal tubular defects, we analyzed the segmental and subcellular expression of ClC-5 in the kidney [6]. Immunoblotting studies identified the $\sim 80-\mathrm{kDa} \mathrm{ClC}-5$ in human kidney cortex and medulla. Immunohistochemistry revealed $\mathrm{ClC}-5$ expression in epithelial cells lining the PT, the thick ascending limb (TAL) of Henle's loop, and the intercalated cells (ICs) of the collecting ducts. Analytical subcellular fractionation of the human kidney suggested that ClC-5 colocalizes with the vacuolar $\mathrm{H}^{+}$-ATPase and is associated with markers of early endosomes (Rab 5a). Confocal microscopy studies using opossum kidney (OK) cells, a model for PT cells, confirmed that $\mathrm{ClC}-5$ colocalized with albumincontaining endocytic vesicles that form part of the receptor-mediated endocytic pathway [6].

Taken together, these studies indicated that the expression of ClC-5 in multiple nephron segments could explain the complex phenotype of Dent's disease. A loss of ClC-5 function in the TAL, which is a major site of regulated calcium reabsorption, may result in hypercalciuria. Since the reabsorption of LMW proteins is restricted to the PT and involves endocytosis with subsequent transport to the acidic vacuolar-lysosomal system, dysfunction of $\mathrm{ClC}-5$ in PT endosomes may impair endosomal acidification and lead to inefficient reabsorption of LMW proteins and dysfunction of PT cells.

\section{Characterization of a CIC-5 Knockout Mouse Model for Dent's Disease}

Dent's disease is thus characterized by PT defects that include LMW proteinuria, and usually aminoaciduria and glycosuria, together with hypercalciuria and nephrocalcinosis. In order to better understand the roles of ClC-5 and the pathophysiology of Dent's disease, we characterized a ClC-5 knockout (KO) mouse obtained by homologous recombination targeting exon VI of Clcn5 [7]. The KO mice were viable at birth and showed normal growth and survival. Northern and Western blot analyses confirmed the absence of ClC-5 in the kidneys of $\mathrm{KO}$ mice. Mice lacking ClC-5 developed renal tubular defects, which included LMW proteinuria, aminoaciduria, glycosuria, phosphaturia and polyuria. They also developed hypercalciuria and nephrocalcinosis, while progressive renal failure was observed with aging. Further investigations indicated that the hypercalciuria in the $\mathrm{ClC}-5 \mathrm{KO}$ mice is of bone and renal origin, and is not caused by increased intestinal calcium absorption - despite elevated levels of $1 \alpha, 25$-dihydroxyvitamin D3 [8].

LMW proteins $(<70 \mathrm{kDa})$ are normally filtered at the glomerulus and avidly recaptured by receptor-mediated endocytosis in PT cells. ClC-5 has been localized to early endosomes belonging to this pathway [6]. In order to study 
this process more closely, mice were injected with peroxidase, a classical endocytic tracer that is filtered by the glomerulus and endocytosed by PT cells. Cytochemistry and electron microscopy showed a severe impairment of protein endocytosis by PT cells in ClC-5 KO mice, so that peroxidase bound to the brush border was poorly transferred into early endocytic vesicles [7]. These data, which were confirmed in another mouse model [9], demonstrated that impairment of receptor-mediated endocytosis in PT cells of Clcn5-deficient mice provides a basis for the defective uptake and increased urinary excretion of LMW proteins.

\section{Loss of CIC-5 Impairs Endocytosis by Defective Trafficking in Kidney Proximal Tubules}

The endocytosis and processing of LMW proteins involve the multiligand tandem receptors, megalin and cubilin, that are abundantly expressed at the brush border of PT cells [10]. Megalin, the largest member of the LDL receptor family, interacts with cubilin and both receptors are internalized together. Diseases of PT cells, collectively called renal Fanconi syndromes, are all characterized by LMW proteinuria. This is reproduced in megalin $\mathrm{KO}$ mice and cubilin-defective dogs [10].

Since progression along the endocytic apparatus depends on endosomal acidification, the defective endocytosis observed in Dent's disease [4] and ClC-5 KO mice $[7,9]$ has been attributed to impaired endosomal acidification secondary to loss of chloride permeability. However, drugs abrogating vacuolar acidification do not affect the rate of endocytic uptake, but inhibit recycling or arrest transfer to lysosomes [11]. We therefore looked for a more specific alteration along the endocytic pathway in Dent's disease, i.e. a lack of the essential components, megalin and cubilin, at the brush border. To this aim, we extensively characterized the endocytic defect in ClC-5 KO mice and found that ligands of both megalin and cubilin were affected. Next, we showed a decrease in total kidney content of megalin and cubilin at the protein level but not at the mRNA level. Finally, using analytical subcellular fractionation and quantitative immunogold labelling, we demonstrated a selective disappearance of megalin and cubilin at the brush border of PT cells. These observations allowed us to conclude that defective protein endocytosis linked to $\mathrm{ClC}-5$ inactivation is due to a major and selective loss of megalin and cubilin at the brush border, reflecting a trafficking defect in renal PT cells [11].

\section{Altered Polarity and Expression of $\mathrm{H}^{+}$-ATPase without Ultrastructural Changes in Kidneys of Dent's Disease Patients}

Our initial studies have shown that ClC-5 colocalizes with the vacuolar $\mathrm{H}^{+}$-ATPase in PT cells and ICs [6]. Studies of $\mathrm{KO}$ mice [7] have established the role of ClC-5 in PT endocytosis, but the consequences of ClC-5 mutations on the human kidney remained unknown.

We have established a multicentric database of renal biopsies from patients with Dent's disease and documented inactivating ClC-5 mutations [12]. Light microscopy revealed a normal renal architecture or glomerulosclerosis, tubular dedifferentiation, and mild interstitial fibrosis. Renal calcifications were identified at all stages. Despite the generalized PT dysfunction, electron microscopy did not reveal any ultrastructural abnormalities in PT cells, and the endocytic apparatus was normal. However, immunohistochemistry studies demonstrated a consistent inversion of $\mathrm{H}^{+}$-ATPase polarity in PT cells to a basolateral distribution contrasting with its apical location in the normal kidney. This inversion of polarity was specific and did not affect distribution of aminopeptidase, megalin or $\mathrm{Na}^{+}-\mathrm{K}^{+}$-ATPase. Furthermore, apical $\mathrm{H}^{+}$-ATPase expression was absent in $\alpha$-type intercalated cells [12]. These modifications in polarity and/or expression of the $\mathrm{H}^{+}-$ ATPase point to an interaction between the C-terminus of $\mathrm{ClC}-5$ and $\mathrm{H}^{+}$-ATPase that would be essential for the proper targeting or stability of the latter. These data are important in the investigation of molecular interactions involving $\mathrm{ClC}-5$ and help us to understand some aspects of Dent's disease, namely the deficit in urinary acidification observed in some patients [3].

\section{Comparative Ontogeny and Segmental Distribution of CIC-5}

The clinical manifestations of Dent's disease often occur during childhood, and LMW proteinuria is a consistent feature of the disease. In particular, the early onset of some cases, in which tubular proteinuria was discovered in the first month of life [13], suggests that segmental expression of $\mathrm{ClC}-5$ in PT must be acquired before birth. We have recently investigated the ontogeny of ClC-5, and its processing and segmental distribution during nephrogenesis [14]. These studies showed that ClC-5 undergoes a rapid induction at an early stage of mouse nephrogenesis, followed by a progressive maturation during late nephrogenesis. Studies with the enzyme PGNase 
F confirmed the early N-linked glycosylation of ClC-5, and the progressive maturation of the core protein during nephrogenesis. The distribution of $\mathrm{ClC}-5$ in PT and $\alpha-$ type IC, and its colocalization with $\mathrm{H}^{+}$-ATPase at these locations, are already achieved at embryonic day (E) 15.5. In human nephrogenesis, $\mathrm{ClC}-5$ is detected early during the second trimester, with a distribution that includes developing PT cells and, later, IC. These results complete early observations that the apical expression of brush border components and the distribution of megalin to the apical clathrin-coated membrane domains and endosomes coincide with the onset of glomerular filtration in rats [15]. The coexpression of $\mathrm{ClC}-5$ and $\mathrm{H}^{+}$-ATPase in PT cells immediately after initiation of glomerular filtration could thus indicate the progressive maturation of renal tubular function and explain the decrease in the concentration of LMW proteins in the amniotic fluid during gestation [16], as well as the early phenotypic variants of Dent's disease [13].

In addition to PT cells, $\mathrm{ClC}-5$ and $\mathrm{H}^{+}$-ATPase are codetected from E15.5 in mouse in the apical area of $\alpha$-type IC [14]. In human nephrogenesis, isolated cells positive for carbonic anhydrase type II (CAII) are identified within ureteric buds and medullary collecting ducts (CDs) at 15 gestation weeks (GW), and rare, isolated cells positive for $\mathrm{H}^{+}$-ATPase are identified at $19 \mathrm{GW}$. In contrast, ClC- 5 could not be identified in IC before $24 \mathrm{GW}$. The colocalization of ClC-5 with CAII and/or $\mathrm{H}^{+}$-ATPase in IC was only observed postnatally, confirming data obtained in mature kidney [6]. These results suggest species differences in the maturation of IC [17] and support the hypothesis that, in humans, the differentiation of IC occurs later than that of the principal cells of the CD [18].

\section{Recent Developments and Perspectives}

Recent developments in the molecular genetics and pathophysiology of Dent's disease should be pointed out. Crucially important information is the demonstration that Dent's disease is genetically heterogeneous. In a careful analysis, Hoopes et al. [19] were unable to find mutations in the coding sequence and promoter regions of CLCN5 in 13 of 32 unrelated patients meeting the clinical criteria for the disease. Furthermore, they excluded linkage to the $C L C N 5$ region in a three-generation family [19]. These data suggest that mutations in genes different from CLCN5 can be involved in Dent's disease. These genes presumably code for proteins that interact, directly or indirectly, with ClC-5. For instance, an interaction between the C-terminus of $\mathrm{ClC}-5$ and cofilin, a protein involved in the depolymerization of actin in the immediate vicinity of endosomes, has been documented in cultured cells of PT origin [20]. This could be crucial for mediating uptake of LMW proteins by PT cells.

In addition to genetic heterogeneity, mutation analysis has provided valuable information relating to the structure of $\mathrm{ClC}-5$. The X-ray crystal structures of two bacterial CLC proteins at 3.0-Å resolution have recently been established [21], confirming the prediction that the CLC channels are homodimers with a double-barreled configuration. Each subunit has its own pore and $17 \alpha$-helices inserted into the plasma membrane, with an anti-parallel orientation that brings together residues responsible for anion selectivity [21]. Based on these data, a model for human $\mathrm{ClC}-5$ has been established, allowing analysis of the role of 15 non-truncating mutations (14 missense and one in-frame deletion) of CLCN5 [22]. This analysis revealed that none of these mutations involves the selectivity filter, but that most of them $(12 / 15)$ are clustered at the interface of the two subunits of the homodimeric channel [22]. The functional evaluation of other naturally occurring mutants is necessary in order to define the role of conserved domains for $\mathrm{ClC}-5$ function and/or trafficking: a case in point is a C-terminus internalization motif, resembling the PY motif that is essential for the internalization and degradation of the epithelial sodium channel, $\mathrm{ENaC}[23]$.

In addition to PT and IC cells, ClC-5 is expressed in the TAL of Henle's loop [6], but its role in this segment remains unclear. However, recent data obtained in a cultured TAL murine cell line, as well as in mice exposed to water deprivation, suggest that exposure to hypertonicity upregulates the expression of $\mathrm{ClC}-5$ at the mRNA and protein levels. Thus, the regulation of ClC-5 expression may be crucial for the adaptation to a hypertonic environment and the preservation of correct endocytic activity/ targeting in TAL cells [24].

A central hypothesis is that $\mathrm{ClC}-5$ provides the chloride conductance necessary for endosomal acidification by the electrogenic vacuolar $\mathrm{H}^{+}$-ATPase $[7,9,25]$. Based on decreased ATP-dependent fluorescence quenching of lowdensity vesicles isolated from $\mathrm{ClC}-5 \mathrm{KO}$ mice loaded in vitro by acridine orange, preliminary data from Gunther et al. [26] support this view. However, these vesicles were not characterized as early endosomes (the well-documented trafficking defect in the PT cells of ClC-5 KO mice may impair their purification and yield contamination by nonendocytic acidified organelles), and the residual quenching in these $\mathrm{KO}$ vesicles still showed chloride dependence 
[26]. In other words, a crucial role of ClC-5 in endosomal acidification remains to be proven.

\section{Conclusions}

The studies outlined here have provided critical information to understand the pathophysiology of Dent's disease, taken as a paradigm for renal Fanconi syndrome and nephrolithiasis. The characterization of the cellular and subcellular distribution of ClC-5 and detailed investigations in the $\mathrm{ClC}-5 \mathrm{KO}$ mouse have demonstrated multiple roles for this chloride channel in the kidney. These studies also provided novel insights into essential functions such as apical endocytosis, handling of proteins by renal tubu- lar cells, calcium metabolism, and urinary acidification. This information and the validation of a mouse model that harbors essential traits such as hypercalciuria and nephrocalcinosis should now prove useful in the development of therapeutic strategies relevant for Dent's disease, renal Fanconi syndromes and kidney stones.

\section{Acknowledgments}

The authors thank R. Beauwens, E.I. Christensen, S. Guggino, W.B. Guggino, T. Igarashi, T. Jentsch, P. Moulin, S. Scheinman, R. Thakker and M.-F. Van Den Hove for support and fruitful discussions. These studies were supported by the Belgian Forton Foundation, FSR (UCL), FNRS and ARC.

\section{References}

1 Coe FL, Parks JH, Asplin JR: The pathogenesis and treatment of kidney stones. N Engl J Med 1992;327:1141-1152.

-2 Scheinman SJ, Pook MA, Wooding C, Pang JT, Frymoyer PA, Thakker RV: Mapping the gene causing $\mathrm{X}$-linked recessive nephrolithiasis to $\mathrm{Xp} 11.22$ by linkage studies. J Clin Invest 1993;91:2351-2357.

-3 Scheinman SJ: X-linked hypercalciuric nephrolithiasis: Clinical syndromes and chloride channel mutations. Kidney Int 1998;53:3-17.

$\checkmark 4$ Lloyd SE, Pearce SHS, Fisher SE, et al: A common molecular basis for three inherited kidney stone diseases. Nature 1996;379:445-449.

$\checkmark 5$ Jentsch TJ, Stein V, Weinreich F, Zdebik AA: Molecular structure and physiological function of chloride channels. Physiol Rev 2002;82: 503-568.

6 Devuyst O, Christie PT, Courtoy PJ, Beauwens R, Thakker RV: Intra-renal and subcellular distribution of the human chloride channel, CLC-5, reveals a pathophysiological basis for Dent's disease. Hum Mol Genet 1999;8:247257.

7 Wang SS, Devuyst O, Courtoy PJ, et al: Mice lacking renal chloride channel, ClC-5, are a model for Dent's disease, a nephrolithiasis disorder associated with defective receptor-mediated endocytosis. Hum Mol Genet 2000;9: 2937-2945.

$>8$ Silva IV, Cebotaru V, Wang H, et al: The ClC5 knockout mouse model of Dent's disease has renal hypercalciuria and increased bone turnover. J Bone Miner Res 2003; 18:615-623.

$\checkmark 9$ Piwon N, Gunther W, Schwake M, Bosl MR, Jentsch TJ: ClC-5 $\mathrm{Cl}^{-}$-channel disruption impairs endocytosis in a mouse model for Dent's disease. Nature 2000;408:369-373.
10 Christensen EI, Birn H: Megalin and cubilin: Multifunctional endocytic receptors. Nat Rev Mol Cell Biol 2002;3:256-266.

11 Christensen EI, Devuyst O, Dom G, et al: Loss of chloride channel ClC-5 impairs endocytosis by defective trafficking of megalin and cubilin in kidney proximal tubules. Proc Natl Acad Sci USA 2003;100:8472-8477.

12 Moulin P, Igarashi T, Van der Smissen P, et al: Altered polarity and expression of $\mathrm{H}^{+}$-ATPase without ultrastructural changes in kidneys of Dent's disease patients. Kidney Int 2003;63: 1285-1295.

13 Bosio M, Bianchi ML, Lloyd SE, Thakker RV: A familial syndrome due to Arg648Stop mutation in the X-linked renal chloride channel gene. Pediatr Nephrol 1999;13:278-283.

14 Jouret F, Igarashi T, Gofflot F, Wilson PD, Karet FE, Thakker RV, Devuyst O: Comparative ontogeny, processing, and segmental distribution of the renal chloride channel, ClC-5. Kidney Int 2004;65:198-208.

15 Biemesderfer D, Dekan G, Aronson P, Farquhar MG: Assembly of distinctive coated pit and microvillar microdomains in the renal brush border. Am J Physiol 1992;262:F55F67.

16 Mussap M, Fanos V, Piccoli A, et al: Low molecular mass proteins and urinary enzymes in amniotic fluid of healthy pregnant women at progressive stages of gestation. Clin Biochem 1996;29:51-56.

17 Kim J, Tisher CC, Madsen KM: Differentiation of intercalated cells in developing rat kidney: An immunohistochemical study. Am J Physiol 1994;266:F977-F990.

18 Devuyst O, Burrow CR, Smith BL, et al: Expression of aquaporins-1 and -2 during nephrogenesis and in autosomal dominant polycystic kidney disease. Am J Physiol 1996;271:F169_ F183.
19 Hoopes RR Jr, Raja KM, Koich A, Hueber P, Reid R, Knohl SJ, Scheinman SJ: Evidence for genetic heterogeneity in Dent's disease. Kidney Int 2004;65:1615-1620.

20 Hryciw DH, Wang Y, Devuyst O, Pollock CA, Poronnik P, Guggino WB: Cofilin interacts with $\mathrm{ClC}-5$ and regulates albumin uptake in proximal tubule cell lines. J Biol Chem 2003; 278:40169-40176.

21 Dutzler R, Campbell EB, Cadene M, Chait BT, MacKinnon R: X-ray structure of a $\mathrm{ClC}$ chloride channel at 3.0 A reveals the molecular basis of anion selectivity. Nature 2002;415:287294.

22 Wu F, Roche P, Christie PT, Loh NY, Reed AA, Esnouf RM, Thakker RV: Related Modeling study of human renal chloride channel (hCLC-5) mutations suggests a structural-functional relationship. Kidney Int 2003;63:14261432.

23 Schwake M, Friedrich T, Jentsch TJ: An internalization signal in $\mathrm{ClC}-5$, an endosomal $\mathrm{Cl}$ channel mutated in Dent's disease. J Biol Chem 2001;276:12049-12054.

24 Pham PC, Devuyst O, Pham PT, Matsumoto N, Shih RN, Jo OD, Yanagawa N, Sun AM: Hypertonicity increases CLC-5 expression in mouse medullary thick ascending limb cells. Am J Physiol Renal Physiol 2004;287:F747F752.

25 Faundez V, Hartzell HC: Intracellular chloride channels: determinants of function in the endosomal pathway. Sci STKE 2004 May 11; 2004(233):re8.

26 Gunther W, Piwon N, Jentsch TJ: The ClC-5 chloride channel knock-out mouse - An animal model for Dent's disease. Pflugers Arch 2003;445:456-462. 